\title{
Mobile Agent architecture for intelligent Product to increase the effectiveness in End of Life Management
}

\author{
Abdelhak BOULAALAM \\ Computer science department \\ Sidi Mohamed Ben AbdEllah \\ University, GRMS2I, LIIAN, \\ FSDM Fez, MOROCCO
}

\author{
El Habib NFAOUI \\ Computer science department \\ Sidi Mohamed Ben AbdEllah \\ University, GRMS2I, LIIAN, FSDM \\ Fez, MOROCCO
}

\author{
Omar El BEQQALI \\ Computer science department \\ Sidi Mohamed Ben AbdEllah \\ University, GRMS2I, LIIAN, FSDM \\ Fez, MOROCCO
}

\begin{abstract}
Sweep the entire product lifecycle information from design to recycling is the great challenge to overcome in manufacturing worldwide. Actually, managing product after-sales information is a key to innovation and launching new products that meet the customers' expectations. To address this objective, the current study proposes architecture for closing the product lifecycle information by using the emerging technologies (PEID) and Mobile Agent software. In this paper we focus on the End of Life (EOL), especially the after-sales stage. To this end, the proposed architecture aims enable product knowledge management (PKM) by gathering and analyzing product-related information after-sales and with the feedback to the previous phases for help actors to decision making process. Furthermore, we aim to enable the product data and knowledge management (PDKM). The proposed architecture uses the advent of hardware and software related to product identification technologies and multi-agent systems (MAS).
\end{abstract}

\section{Keywords}

Product Lifecycle Management (PLM), Multi-agent systems (MAS), Radio Frequency Identification (RFID), End of Life, Change Management, Knowledge Management.

\section{INTRODUCTION}

A sustaining new product development capability is the key factor to improve competitiveness for enterprises in the market management [5]. Product lifecycle management (PLM) is the further expand of Product data management (PDM); its objective is to provide more product-related information to the extended enterprise over whole product life cycle. PLM allows the innovation of enterprise operations by integrating people, processes, business systems, and information throughout product life cycle and across extended enterprise. It integrates information technology, modern management technology, advanced manufacturing technology which are used during all product life cycle phases. It allows a better management the information, processes and resources of product life cycle. The market adaptability and competitive capability [5] are possible within the integration and optimization operation of substance flow, information flow and value flow. According to CIMdata [1], PLM is a strategic business approach that applies a consistent set of business solutions as support of the collaborative creation, management, dissemination, and use of product definition information across the extended enterprise and spanning from product concept to end of life, integrating people, processes, business systems, and information. Moreover, according to A. Saaksvuori [4], a PLM system is a collaborative backbone allowing people throughout extended enterprises to work together more effectively.
The theory of a product life cycle was first introduced in the 1950s to explain the expected life cycle of a typical product from design to obsolescence phase. Carole Hedden [17] observed that the cycle is represented by a curve that can be divided into four distinct phases: introduction, growth, maturity, and decline. The main goal is to maximize the product's value and profitability at each stage. It could be considered as a marketing theory. Generally, in the PLM domains, the product lifecycle consists of three main phases:

- Beginning of life (BOL) including design and production;

- Middle of life (MOL), including logistics (distribution), use, service, and maintenance;

- End of life (EOL), including reverse logistics (collecting), remanufacturing (disassembly, refurbishment, reassembly, etc.), reuse, recycle, and disposal.

In addition, the focus today in the business environment involves mainly innovation: products that differentiate themselves from others while also being affordable, reliable and introduced early in market phase. Total management of the product lifecycle is critical to innovatively meet customer needs throughout the entire life cycle without driving up costs, sacrificing quality, or delaying product delivery [3].

In spite of its objective, PLM has not received much attention until so far from industry because there are few efficient tools to gather product life cycle data after product sales.

Although there have been many previous works about product lifecycle management in the beginning of life (BOL) and middle of life (MOL) phases, few addressed the system architecture for PLM, in particular, the end of life (EOL) phase and the closed-loop PLM in which information and activities have closed-loop flows due to the information visibility.

The combination of the four biological phases (introduction, growth, maturity, and decline) and the three PLM phases and its management in terms of cost optimization, quality and deadline constitute the challenge to overcoming for each enterprise to survive. However, the management of the data product in intelligent way represents the backbone for innovation to satisfy the final clients. For this fact, the notion of intelligent product is introduced.

In this paper, firstly we give the recent definitions related to the paradigms HMS (Holonic Manufacturing system), the Avatar Product and the Intelligent Product. These paradigms are studied and analyzed regarding their intelligent aspect. Secondly we aim to investigate the product lifecycle 
management as a problem of traceability, decision making and intelligent dimension, trying to establish a new way of thinking for managing product data along the lifecycle, especially in end of life stage. In particular, the paper aims to present the new architecture by adding the over layer product lifecycle for increasing the effectiveness in the end of life, based on the concept of intelligent product services.

The paper is organized as follows: section 2 presents the literature review of the intelligent product topic. Section 3 presents some related research works and some recent projects. In Section 4, we present our proposed architecture based on radio frequency identification (RFID) and Mobile Agent technologies. Finally, Section 5 provides some concluding remarks and future work.

\section{BACKGOUND REVIEW}

This section gives some relevant definitions of previous "intelligent products" such as the product avatar, product Holon, and finally the recent definition of Intelligent Product paradigm; by presenting the related research works involving the product in the heart of the system. Researches related to intelligent products have also been developed, on which the idea is connecting the physical products with their counterparts within information systems.

Hribernik et al. in [6] present the concept of (semi-) autonomous and product item centric information management based on the Avatar concept. This vision is illustrated in the figure 1 and figures 2 , the comparison of the traditional and a new approach is also given.

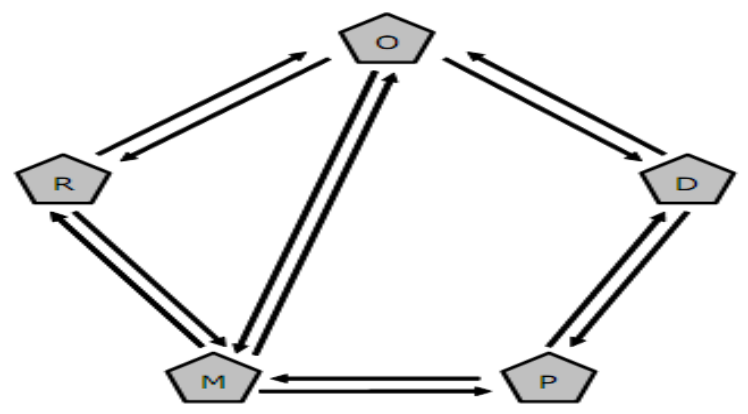

Fig 1: Traditional approach

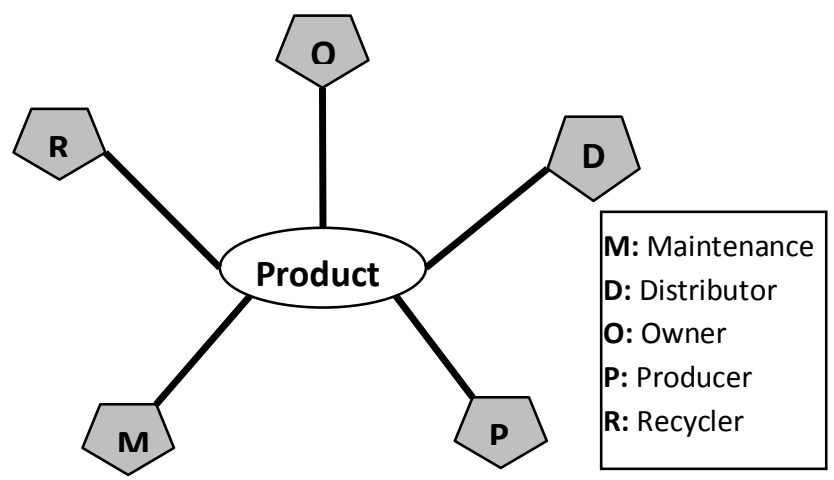

Fig 2: New approach (AVATAR)

We can assume the benefits of this approach in two points:

- The first is that the product in the key component of the network; it is also the unique participant in the network having communication to each other actors during life-cycle in simultaneous way.
- The second one is the reduced amount of information links compared to the traditional perspective.

In other hand this proposition is based on the same research area by McFarlane [7], C.Y Wong [8], and M. Kärkkäinen [9]. Furthermore, J. Cassina approach is based on product avatar aiming to define new business opportunities through the concept of Extended Product Lifecycle Management (ExtPLM) [10]. They assume that this strategy allows more efficiency management among all product lifecycle phases.

The other most important paradigm is the Holon (Holonic Manufacturing Systems). Holonic Manufacturing based on the concept of "holonic systems", developed by Koestler [14]. Koestler proposed holon, holarchy and holonic system to describe the systems that Herbert Simon identified as necessary [29].According to Shen [11] there are many projects in relation with the notion of Holon and their application to satisfy the needs such as interoperability, distributed control and collaboration between software, hardware, human, and organizational systems.

To assure the coordination between the real and the virtual world, a product oriented modeling concept: holons for systems synchronization and interoperability are proposed in [12], S. Baïna et al. focus on enterprise interoperability issues. A Holon based approach is defined in order to synchronize views in the business world and in the physical manufacturing world using the Holon concept.

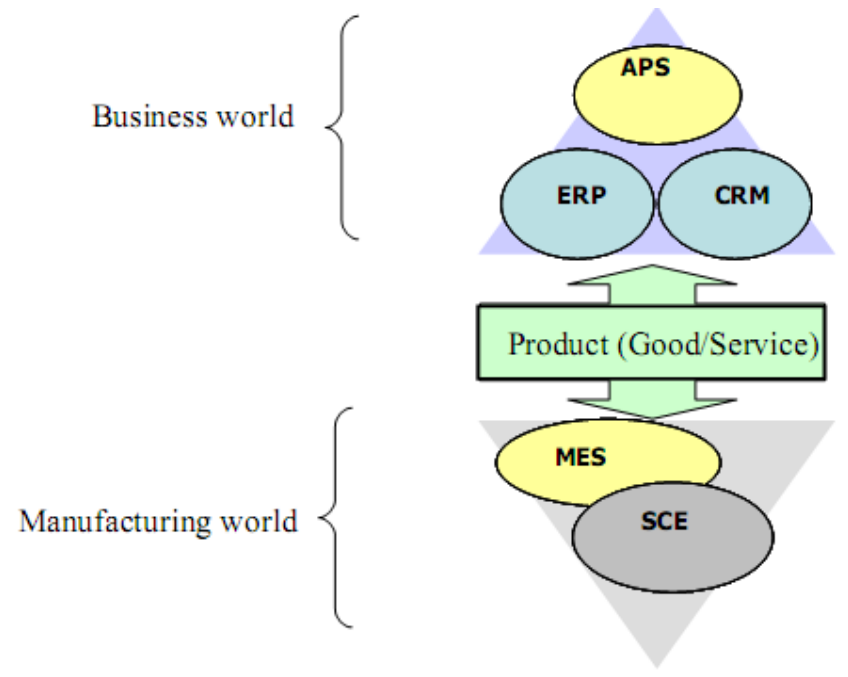

Fig 3: Product centric approach

The general architecture of the Holon is showed in the figure bellow. 


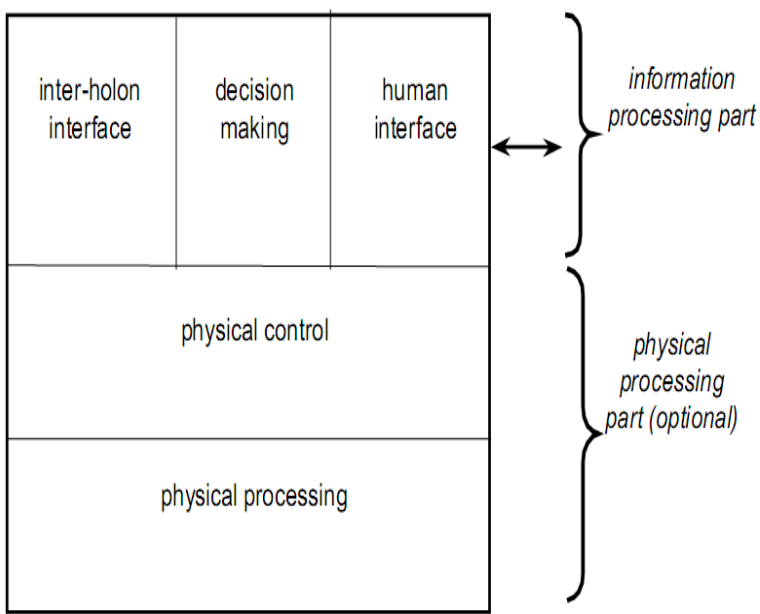

Fig 4: General Architecture of a Holon [13]

The physical processing layer is the actual hardware performing the manufacturing operation like milling or assembly. It is controlled by the physical control layer. The decision making represents the kernel of the Holon and provides two interfaces: the first for interaction with other holons, and the second for interaction with humans. Furthermore, a holarchy is a hierarchy of self-regulating holons, which function (a) as autonomous wholes in supraordination to their parts, (b) as dependent parts in subordination to controls on higher levels, and (c) in coordination with their local environment. Furthermore, Terzi et al. [16] proposed a metamodel for the traceability of products over the lifecycle, which is a basis on the holonic approach for fostering interoperability along diverse enterprise applications.

The market increasingly demands products that are customized, yet available with short delivery times. Consequently, the business focus must shift from designing and selling physical products, to supplying a system of products and services ('product/services' or 'extended products') that are jointly capable of fulfilling users' demands, while also reducing total life-cycle costs and environmental impacts. Agent technology is used to implement HMS applications because the two approaches are almost similar. By comparison holons and agents are very similar concepts (for a detailed comparison of these two notions see [15]).

After introducing the concept of Holon product and Avatar product, the rest of this section will be reserved for describing and analyzing the latest definitions and research on intelligent product found in the literature.

Wong et al defined an intelligent product as a physical and information based representation that [8]:

- possesses a unique identification

- $\quad$ is capable of communicating effectively with its environment

- can retain or store data about itself

- deploys a language to display its features and requirements
- is capable of participating in or making decisions relevant to its own destiny

Through this state awareness, the intelligent product has the ability to make appropriate decisions and trigger the actuation of that decision. Figure 5 summarize the McFarlane et al. definition of Intelligent Product [7], the three important points of this representation include:

- the physical product(jar of spaghetti sauce)

- the information-based representation of the product(database)

- the intelligence is provided by the decision making agent

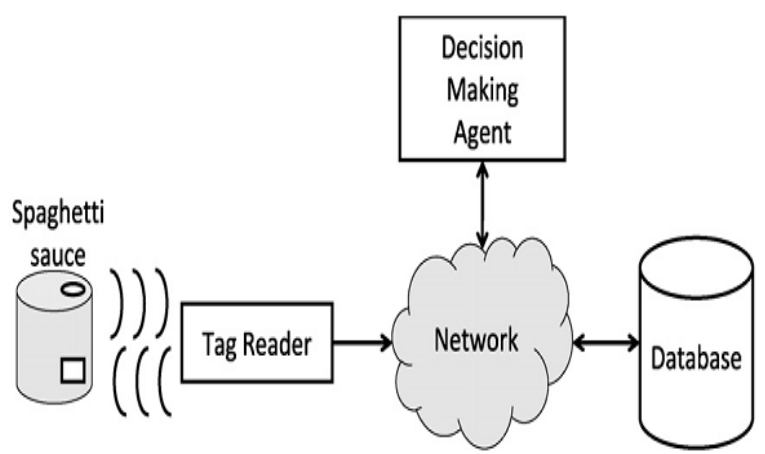

Fig 5: Intelligent jar of spaghetti sauce

The use of intelligent products gains popularity as the accuracy of information held in databases increasingly mismatches reality due to increasing product variety and complexity in supply networks [9].

The intelligent product concept has also been used in eMaintenance. By leveraging Internet technologies, the emaintenance system provides a product's user with the capability to remotely link to the manufacturer through the Internet. The DYNAMITE project looks to develop sensing, monitoring and communication technologies to enable global eMaintenance [31]. Smart tags incorporate unique identity and health history; mobile devices are used to access to central decision guidance systems which allow scheduling of maintenance through cost optimisation.

Bussmann and Sieverding [32] developed a holonic system for existing engine assembly systems in the automotive industry that offered robustness and scalability as additional resources can be added easily to the system, resulting in promising achievements in existing assembly systems.

P. Valckenaers et al. [30] define an intelligent product as: Intelligent product $=$ "intelligent being + intelligent agent"' He presents the concept of intelligent beings as a vehicle to achieve suitability for integration. Figure 6 summarize all internal components of an Intelligent Product proposed by Valckenaers. 


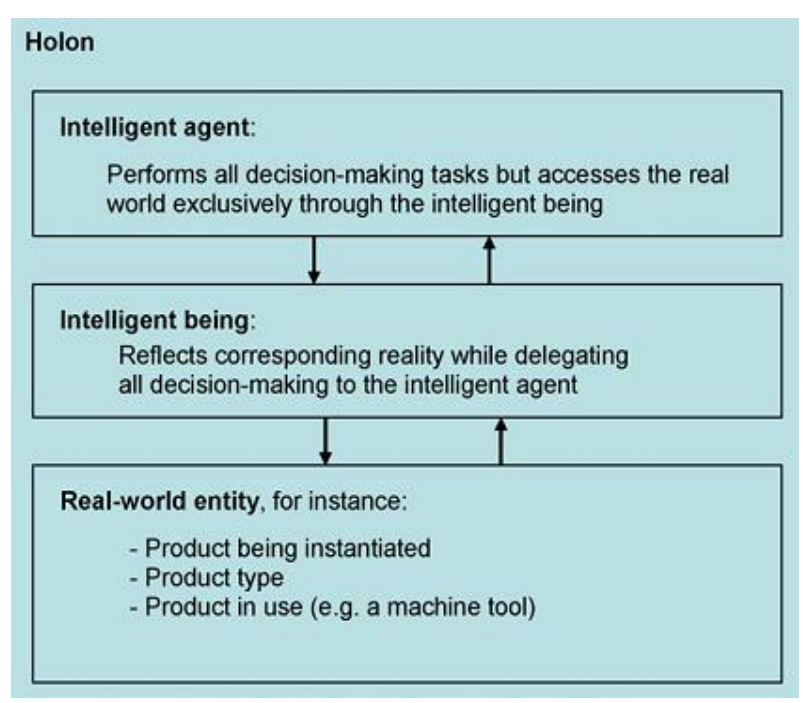

Fig 6: Internal structure of an intelligent product [30]

The other application of intelligent product is the Internet of things (IoT). Kiritsis [19] introduce a new definition of the notion of Intelligent Product inspired by what happens in nature with us as human beings and the way we develop intelligence and knowledge. He defines four levels classification of intelligence. The first one, the physical products without any embedded system (no interaction with environment). The second level, the physical products with embedded simple sensors. The third level is that the physical products with embedded sensors, memory and data processing capabilities. Finally the level when the physicals products with Product Embedded Information Devices (PEID), the identification and communication capabilities are now added at this level.

The same authors in [2] proposed an Intelligent Product architecture, which is based on the next postulate - the product is an actor who manages its evolution in cooperation with the various actors of the life cycle, equipped with Intelligent Data Unit using Radio Frequency Identification (RFID), and the Mobile Agent.

After discussing the Intelligent Product paradigm, we summarize that this is a new paradigm in the way that it is possible to monitor each single item of a product type. In fact, the focus is to collect information for the next version of a product from the product lifecycle phases, especially the end of life phase. The gathered data can be analyzed and transformed from raw information to knowledge which can then be used to optimize the first phase of biological product lifecycle (lunch phase). For this, the product embedded identifications systems combined with the mobile agent can be used to bridge the last mile between the physical product and the PLM system solutions and can remove the manual errors and delay in information sharing in extended enterprise environment.

Although various definitions of intelligent product have been proposed, we propose Mobile Agent architecture for intelligent Product to increase the effectiveness in the End of Life motivated by the way we develop intelligence and knowledge. For this, the next section describes the technologies behind the Intelligent Product paradigm.

\section{INTELLIGENT PRODUCT AND TECHNOLOGIES BEHIND}

Our proposed architecture is based on two technologies, the Product Embedded Identification (PEID) and the Mobile Agents. Firstly, we discuss the Product Embedded Identification paradigm, especially the RFID system architecture for closing the product lifecycle and gathering product data. At last, the Mobile Agents approach is discussed within the extended enterprise environment.

\subsection{Product Embedded Information Devices}

Recent advances in miniaturization of computing devices, communications and digital storage technologies, along with the new data-acquisition and auto-identification technologies capacity, have enabled gathering and storing of heterogeneous product data among all product phases.

In recent years automatic identification procedures (Auto-ID) have become very popular in many service industries, purchasing and distribution logistics, industry, manufacturing companies and material flow systems. Automatic identification procedures provide information about people, animals, goods and products in transit phase [21].

There are several technologies to identity product .In this section, the study is focused on RFID system. Radio frequency identification (RFID) uses radio waves to transmit the information from an RFID tag affixed to the physical object. A brief overview of different automatic ID systems with similar functions as RFID is shown in Figure 7.

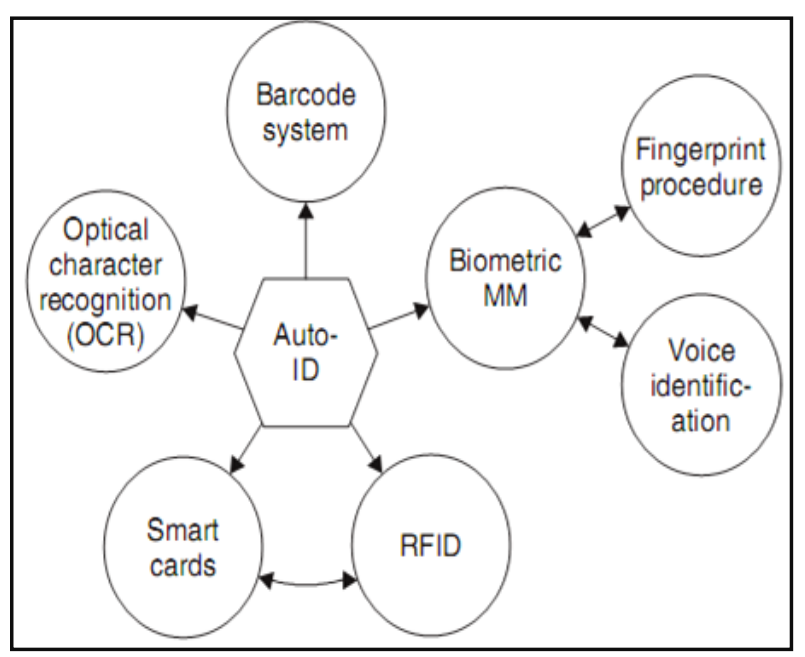

Fig 7: Overview of the most important auto-ID procedures

The emerging product identification technologies such as radio frequency identification (RFID), various kinds of sensors, and AUTO-ID enable gathering product lifecycle data (e.g. identification, location, product status, etc.), which leads to the closed loops of product lifecycle data and information flows over the whole product lifecycle.

During product lifecycle, the data will be gathered periodically or based on events triggering at various operational sources of different lifecycle phases. In addition to this, as enterprises become increasingly global and networked (virtual enterprise), product lifecycle data tends to become spread on computer systems of multiple companies. 


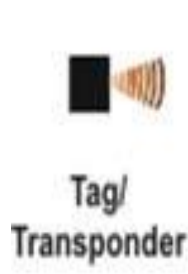

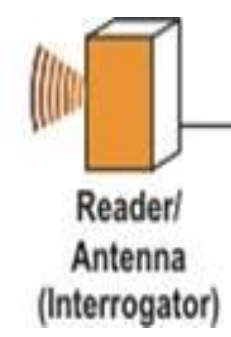

Fig 8: A typical RFID system

As shown in previous figure 8; an RFID system is constituted by:

- $\quad$ an RFID device (tag);

- a tag reader with an antenna and transceiver; and

- a host system or connection to an enterprise system.

While the initial RFID application areas were generally industrial, applications like retail sector, supply chain management, warehouse management, logistics, manufacturing, military applications, and service sector also present potential applications for RFID technologies [20].

The interest in RFID is also reflected in various fields and projects of research in the academic community such as innovation management, project management, SCM, PLM, IS, eCommerce, etc. For instance, Vrba et al. [27] propose special RFID agents as mediators between physical RFID readers and other agents. Furthermore, the authors summarize their contribution in four points, where the most important are: introduction of the work piece agents representing physical products that are able-with the help of RFID - to negotiate with transportation agents and other resource agents about the details of production.
In other hand, different project applied the radio frequency identification technology in the Internet of Things such as cluster of European RFID projects (CERP). The Cluster of European Research projects on the Internet of Things CERP-IoT - comprises around 30 major research initiatives, platforms and networks working in the field of identification technologies such as Radio Frequency Identification and in what could become tomorrow an Internet-connected and interconnected world of objects. The projects involved in CERPIoT [27] are listed in the following table (the list is not exhaustive).

The use for RFID in all previous projects is focused on track and trace physical product either the real time control and manage the supply chain. Furthermore, PROMISE project is corresponding widely to our approach; it firstly focuses on the complete product lifecycle from the Beginning of Life (BOL) to the End of Life (EOL) with more emphasis in tracking and managing of information at the last two phases of the product's life cycle. And secondly the closed-loop PLM is the main objective of the PROMISE project.

We resume the use of radio frequency identification in product lifecycle management area in the:

- Requirement for product-oriented information management approach

- Tight coupling between physical items and sub components and the information held about them

- Ability to retrieve information in a simple and timely manner

- The tree previous points in the fundamental elements to enabling Intelligent Product ( the feedback point of view)

Table 1. Projects involved in CERP-IoT

AMI-4-SME [37]

\section{ASPIRE [38]}

BRIDGE [39]

CASAGRAS [40]

\section{CuteLoop [41]}

FIA/RWI [42]

IMS2020 [43]

PROMISE [44]

GRIFS [45]

SMMART [46]
Ambient Intelligence Technology for Systemic Innovation in Manufacturing SMEs

Advanced Sensors and lightweight Programmable middleware for Innovative RFID Enterprise applications

Building Radio frequency Identification solutions for the Global Environment

Coordination and Support Action (CSA) for Global RFID-related Activities and Standardization

Customer in the Loop: Using Networked Devices enabled Intelligence for Proactive Customers Integration as Drivers of Integrated Enterprise

Future Internet Assembly: Real World Internet

Intelligent Manufacturing System 2020

Product orientated manufacturing systems including RFID technology

Global RFID Interoperability Forum for Standards

System for Mobile Maintenance Accessible in Real Time
We note that these projects combine many technologies for developing new approach and philosophies to satisfy their clients. In our research we use the RFID-MAS combination to solve new business issues. RFID are used to bridge the last mile between the product and the PLM solutions and this can remove the manual errors and delay in information sharing.
RFID tags are used for storing the tag identification number. when RFID readers read this ID number, this can be directly sent to our interface layer and activate the product agent artifact to get whatever information needed as well as to filter, store and create the information/knowledge in PLM system. 


\subsection{Multi-agent systems: the mobile agent}

Multi-agent systems are a new paradigm for understanding and building distributed systems, where it is assumed that the computational components are autonomous: able to control their own behavior in the furtherance of their own goals.

The Mobile agent is a kind of software. It is autonomous, and it migrates from one host to another in diverse network environments [22], and can be used in distributed architecture for the decision-making process [23]. Gray et al[36] gives the following definition of a mobile agent: "Mobile agents are programs that can move through a network under their own control, migrating from host to host and interacting with other agents and resources on each". Furthermore, a mobile agent must have the five following properties [24]:

(1) It should be able to achieve one or more goals automatically.

(2) It should be able to clone and propagate itself.

(3) It should be able to collaborate and communicate with other software and agents.

(4) It must have a scope of competence.

(5) It should have some evolution states to record the computation status.

Various research works have been done around the Mobile Agent concept. C-J Su et all [33] implement a distributed information infrastructure-MADIP by using Intelligent Agent paradigm in medical field, which is able to notify the responsible care-provider of abnormality automatically, offer distance medical advice, and perform continuous health monitoring for those who need it. Furthermore, Damianos Gavalas et al [34] propose the mobile agent (MA) technology for the management of networks and distributed systems as an answer to the scalability problems of the centralized paradigm.

Timon C. Du et al [35] propose a framework for using mobile agents to demonstrate autonomous behavior in the electronic marketplace.

The components of Mobile Agent model used in our research are illustrated in figure 9 .

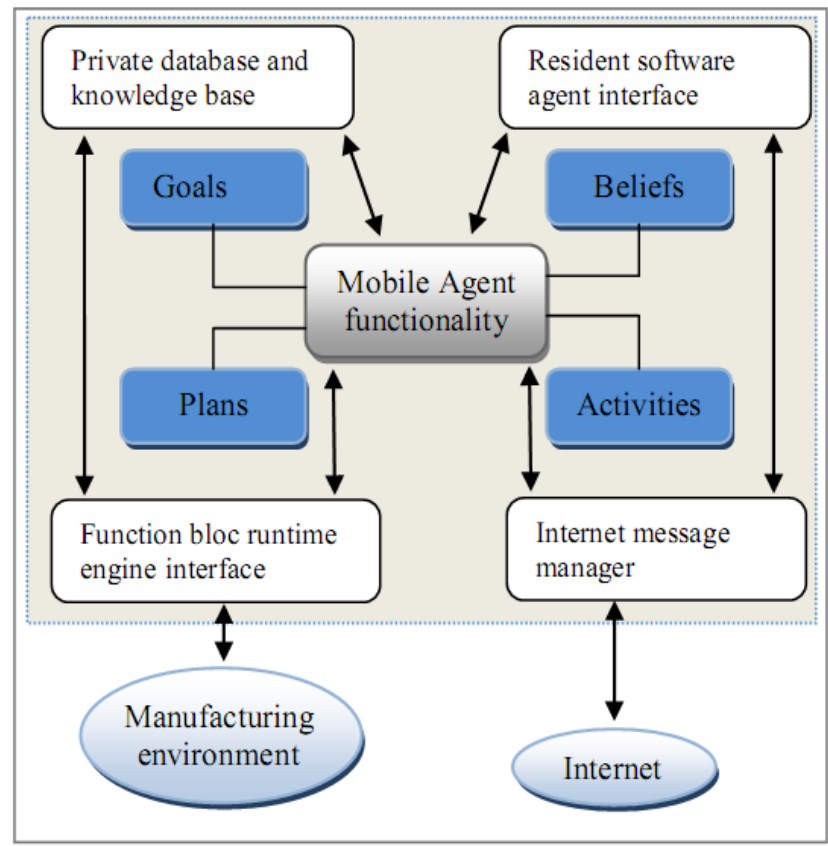

Fig 9: Mobile Agent functionalities

To confront the issues of interoperability, scalability, and openness in heterogeneous environments, a FIPA2000 standard compliant agent development platform-JADE (Java Agent DEvelopment Framework) was adopted for the design and implementation of the proposed architecture. JADE define the mobile agent as entities which are able to migrate or duplicate themselves across multiple network hosts.

In the literature there are many available mobile agent systems (frameworks) for developing mobile agent. Table 2 summarizes the main projects used mobile agent systems for research and applications fields. Furthermore, Java Agent Development framework (JADE Framework) [26] is also used to develop agent applications in compliance with the Foundation for Intelligent Physical Agents (FIPA) specifications.

Table 2. List of commonly used mobile agents [25]

\begin{tabular}{l|l|l|l|}
\hline name & developer & Language & Application \\
\hline Agent Tcl & R. Gray, U Dart. & Tcl Tk & Information management \\
\hline AgentSpace & Ichiro Sato, O. U. & Java & General purpose \\
\hline AgentSpace & Alberto Sylva & Java & Support for dynamic and dist. Appl. \\
\hline Aglet & IBM, Tokyo & Java & Internet \\
\hline Ajanta & Minoseta U. & Java & General purpose \\
\hline Ara & U Kaiserslautern & C/C++, Tcl, Java & Partially connected c. D.D.B. \\
\hline Concordia & Mitsubishi E.I.T. & Java & Mobile computing, Data base \\
\hline JATlite & Standford U. & Java & Information retrieval, Interface agent \\
\hline Kafka & Fujitsu Lab. Japan & Java, UNIX-based & General purpose \\
\hline Kali Scheme & NEC Research I. & Scheme & Distributed data mining, load balancing \\
\hline Knowbots & CNRI & Python & Distributed systems/Internet \\
\hline Messengers & UCI & C (Messenger-C) & General purpose \\
\hline MOA & OpenGroup, UK & Java & General purpose \\
\hline Mole & Stuttgart U. Germany & Java, UNIX-based & General purpose \\
\hline OAA & SRI International, AI & C, C-Lisp, Java, VB & General purpose \\
\hline Odyssey & General Magic & Telescript & Electronic commerce \\
\hline
\end{tabular}




\begin{tabular}{|l|l|l|l|}
\hline Plangent & Toshiba Corporation & Java & Intelligent tasks \\
\hline Tacoma & Norway \& Cornell & C, UNIX-based, & Client/Server model issues/OS support \\
\hline The Tube & David Halls, UK & Scheme & Remote execution of scheme \\
\hline Voyager & ObjectSpace & Java & Support for agent systems \\
\hline
\end{tabular}

After discussing the concepts of Intelligent Product and the technologies behind, the next section focus on our proposed approach, and its contribution in Product Lifecycle Management (PLM).

\section{PROPOSED ARCHITECTURE}

This section focuses on our proposed model based on our related works. In [2] we consider the mutation of the "simple" physical product to an "ambient" product capable to communicate with the others players throughout its entire lifecycle, with the property of having the 'inside-out' control. The focus is on managing the End of Life phase based on the Intelligent Product paradigm.

\subsection{General architecture}

The main advantage of this architecture is the flexibility provided to the enterprise. Our principle guideline is illustrated in the Figure below (Figure 10):

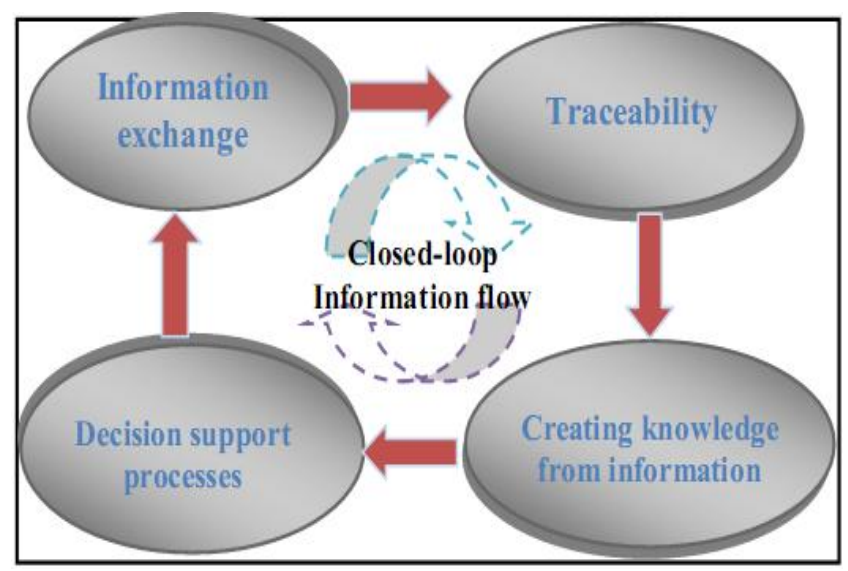

Fig 10: Our approach

The complete architecture is shown in Figure 11. This architecture is based on Agent's software that can be mobile in the case of the extended enterprise, which there are several modules and actors. As illustrated in the figure, in the case of the assembly enterprise, suppliers are geographically distributed. Each physical product has its artifact Mobile Agent (ProductAgent), the first in the real world; the second reside in the system with the possible migration for each site for the tracking process. The second scenario focuses on EOL phase. Otherwise, it is the backbone of our approach; first the EOL is composed of several phases or sub-module; we focus on the after-sales when the product is at the end user. After collecting related data product step, our Agent system based on agent ID product creates/activates the agent at the same identification as the physical product (product agent artifact); this agent can autonomously create another agents corresponding to the other lifecycle module such as CustomerAgent, PlanningAgent, PKAgent etc. The information collected is treated and filtered, in order to building the Product Knowledge (PK), the process of this transformation is monitoring by our Agents. Build a DBKM database is a very important gain for the company that considers its product data a real capital. Based on this knowledge the company can launch new products to market that meet increased customer requirements.

By the use of Mobile Agent, we aim to provide the flexibility and interactivity in the real-time between the PLM players (intra and extra-actors) and the product; which we consider the main actor in product lifecycle. In that case, this interaction will be between the physical product and its counterpart in the system PA (ProductAgent). Technically this data exchange is done through PIED technology.

Another important scenario in this architecture is traceability, generated by change and configuration management. Traceability can be divided into two different areas: the traceability of the product process and the traceability of the order-delivery process. In the product process, traceability is concerned with the planning of the generic product, its creation process and the tracking of the actual development process. Traceability in the order-delivery process is about the tracking of an individual product unit's production and delivery to the customer. The main objective of our approach is to support engineer's designer and others actors of lifecycle for decision making about design improvements and maintenance/service operations by gathering and managing the product data especially in the end of life phase (EOL). The ProductAgent create others agents such as ImpactCalculatorAgent and PKAgent (product knowledge agent) to establish the product knowledge. Products will send back much more information to their manufacturers. It will be used to develop new generations of product.

For the change management module, we introduce the ImpactCalculatorAgent capable to calculate the impact for each proposed change. In other hand, the benefits are: the first help designer, the second is the ability to propose the adequate change. Furthermore, EOL information could go back easily to designers and engineers for the improvement of BOL decisions. Feedback about the use of one generation of a product helps improve future generations. Products that have reached the end of their life are disassembled, and some parts are reused in the start-of-life of new products. To conclude our architecture allows a closed-loop PLM by integrating the EOL phase.

More descriptions of Product Knowledge process are provided in the next section. 


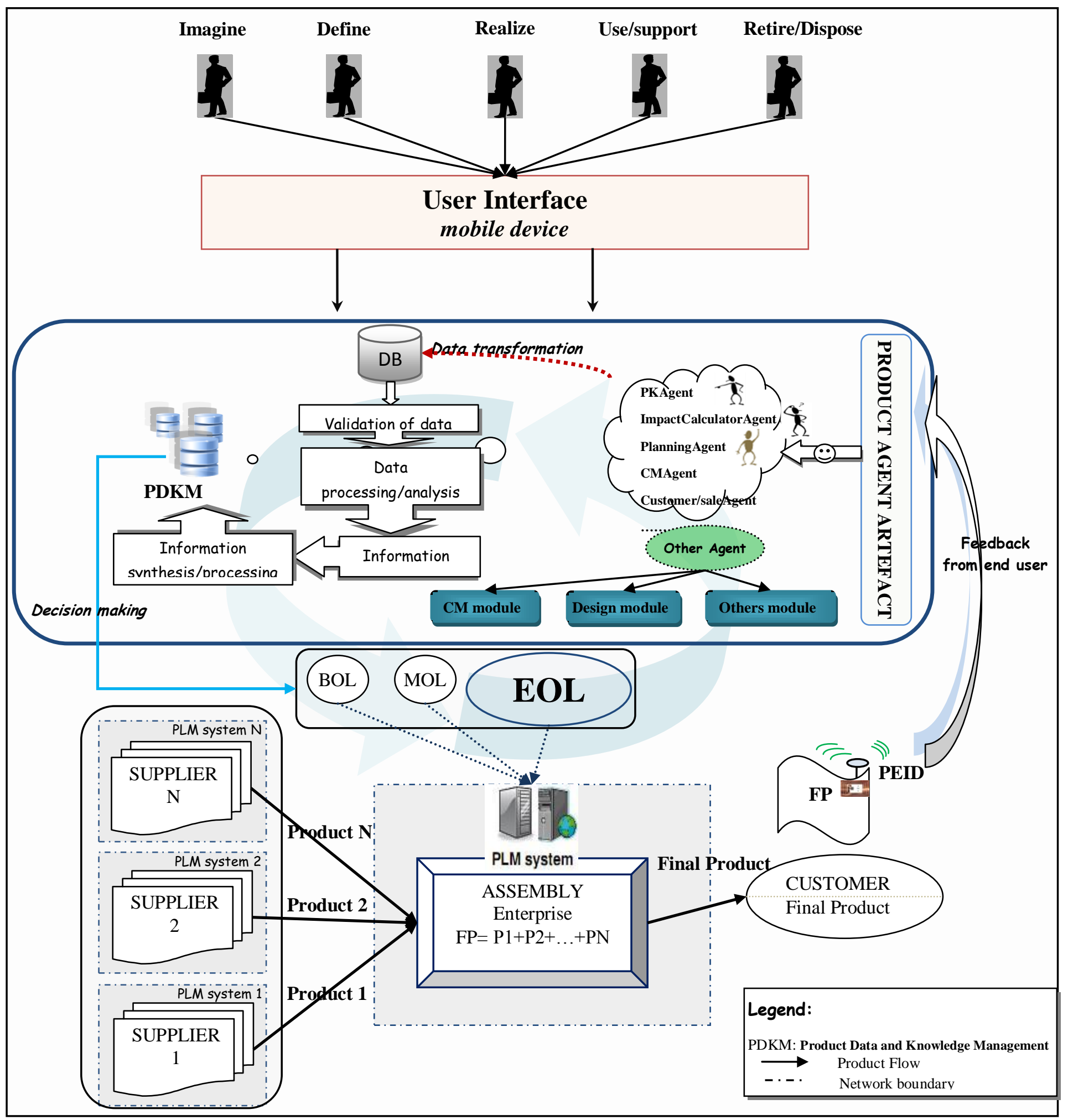

Fig 11: Proposed architecture

\subsection{Product Data and Knowledge Management}

In this section we describe the PK system architecture. The Knowledge part of Knowledge Management includes knowledge of anything about a company, such as its customers, its products, its competitors and its partners. It could be knowledge from the past, current knowledge or, perhaps most valuable, future knowledge. The focus in this part is on the product knowledge creation process in the end of life stage to build more effectiveness in lunch phase. Furthermore, we based on PROMISE product data and knowledge management shown in figure bellow: 

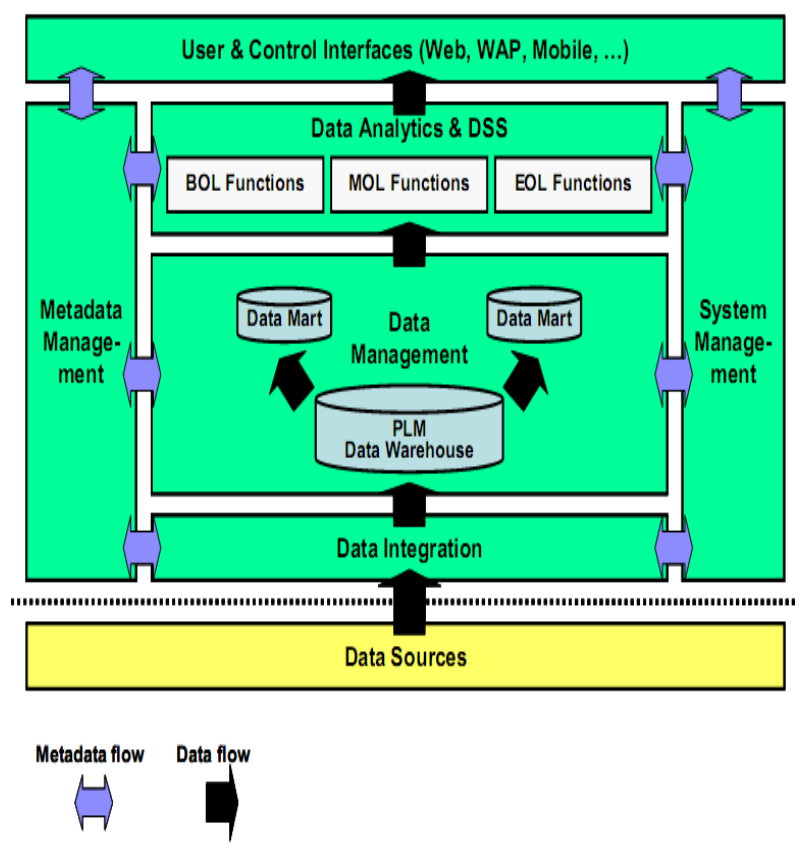

Fig 12: Architecture overview of the PDKM system [18]

Figure 12 illustrates the overall PDKM architecture with its components and the data sources are not a part of the PDKM but feed it with product lifecycle data.

Data Management Layer aims at providing an applicationindependent semantic view of data. In particular, all relevant data are kept in a central database, PLM Data Warehouse, which implements the global schema thereby providing a uniform data representation for queries and analysis. For supporting specific analysis tasks, usually only subsets of the data warehouse are useful, which can be provided in preformed views, the so-called data marts.

In order to validate our approach we add the other layer called DAAgent (Data Analytics Agent), this layer comprises several agents and algorithms to support decision making in order to minimize the product lunch phase and support the other PLM actors.

The proposed approach for gathering data and create the knowledge in distributed environment is shown in figure 13.

This figure describes the general architecture and scenario for creating the knowledge based on the End of Life phase. The main objective is that the product will send back much more information to their manufacturer; we divide the knowledge into two major categories: (a) the first one is the explicit information/knowledge returned by the customer by an interactive participation; the product should offer some services such as the detailed operations for new product etc. The customer could be informed that some components are need to be replaced otherwise the product will have many failures. The direct involvement of the customer provides them access to the suitable information, and provides useful information to the manufacturer. (b) The second category is the tacit information/knowledge. Based on the RFID technologies, the detailed information about the product such as the use of product, the environment effects information, and the product failure details can be automatically collected. After this, the ProductAgent based on CustomerAgent, DAAgent and PKAgent feed the local knowledge repository.

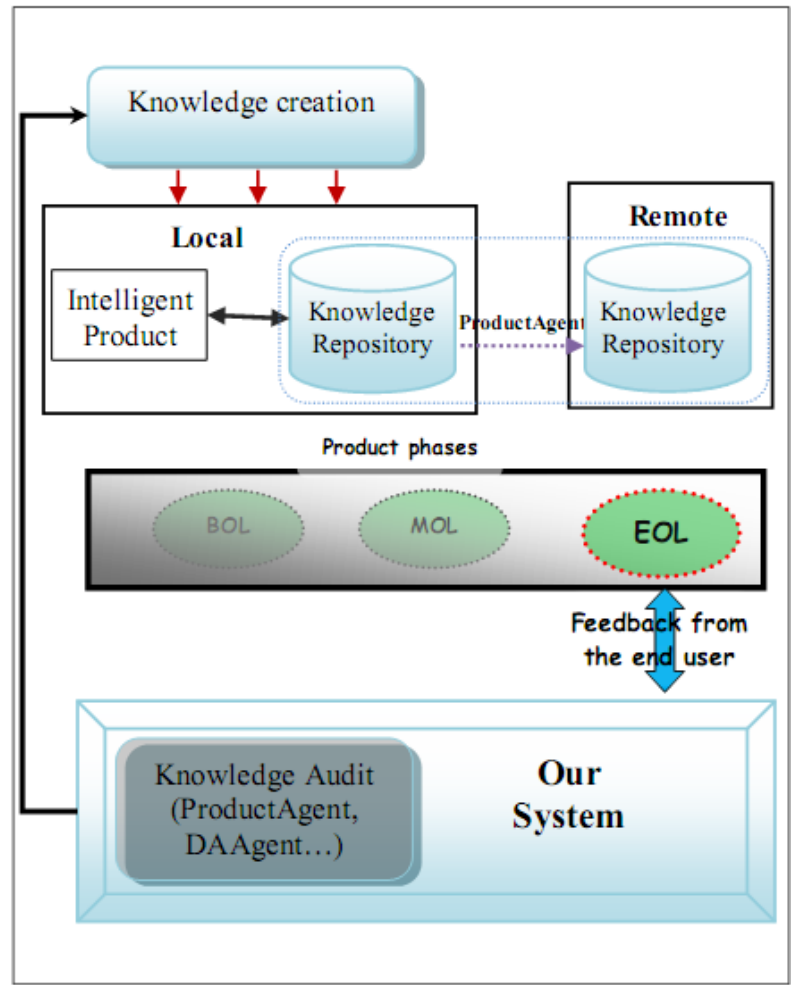

Fig 13: our proposed model for PDKM

On the other hand, like that traceability is a major factor in managing the information asset of enterprise (PME). We divide traceability into two categories. We call the first Geographical traceability; it means the trace and track that travel product from the first company to the final destination. This traceability is guaranteed by the itineraries historic of ProductAgent. The second one is due to the change and configuration management which is more complicated than the first one because of the important interventions on the product. Changes are unavoidable and almost all changes require cost but through the ImpactCalculatorAgent and ProductAgent of global change management module, and through the improved visibility and communication of change information the necessity and impacts of changes can be limited.

Such architecture can easily be extended to an inter-enterprise environment: within this context, data gathered from RFID in the End of Life phase tags are filtered and shared among enterprises using several agents controlled by the MAS framework. Figure 13 shows how information are read from readers (EOL), transformed by the sub-agents of ProductAgent to the knowledge in the local database and distributed outside the enterprise. Finally, our proposed architecture aims to enabling a company to grow revenues by improving innovation, reducing time-to-market for new products, and providing new services for existing products.

\section{DISCUSSION AND CONCLUSION}

Closed-Loop PLM extends PLM in order to close the loop of the information flow among the different lifecycle phases. The closed-loop PLM system provides opportunities to reduce the inefficiency of life cycle operations and gain competitiveness. To this end, this study has proposed an system architecture for closed-loop PLM. This Mobile Agent architecture for intelligent Product to increase the effectiveness in End of Life Management aimed to gather 
product life cycle data during all product life cycle phases, especially the end of life phase. The technologies behind our proposed architecture such as mobile agent and product embedded identification have been introduced. In addition, to streamline several product life cycle operations based on the proposed architecture, possible business scenarios have been introduced.

With the general proposed architecture and the product data and knowledge model, we can get several benefits as follows. First, it can increase customer satisfaction by quickly responding to not only their requests but also environmental variations. Moreover, involving customers is more efficient than just" listening to customers". Second, in the closed-loop PLM, a lot of product life cycle data can be accumulated and used for not only current product life cycle but also next product life cycle.

RFID and Mobile Agent provide a good overview in product end of life phase management. We plan to validate our prototype on the basis of a real case study.

\section{REFERENCES}

[1] CIMdata Inc., The CIMdata PLM Market Analysis Report Series, 2011.

[2] A. Boulaalam, E. Nfaoui, O. EL Beqqali, Mobile Agent PLM Architecture for extended enterprise, IJCSI International Journal of Computer Science Issues, Vol. 8, Issue 1, January 2011.

[3] D. Kiritsis, Ubiquitous product lifecycle management using product embedded information devices, in: Proceedings of IMIS'04, 2004.

[4] A. Saaksvuori, A. Immonen, Product Lifecycle Management, Third Edition, Springer, 2008.

[5] X. Xin-sheng, F. Shui-liang, G. Xin-jian, A Framework for Product Lifecycle Management System, Management Science and Engineering, 2006. ICMSE '06. 2006

[6] K. A. Hribernik, L. Rabe, J. Schumacher, K-D Thoben, The Product Avatar as a Product-Instance-Centric Information Management Concept, International Journal of Product Lifecycle Management, Vol. 1, No. 4, pp.367379.

[7] D. McFarlane, S. Sarma, J. L. Chirn, C.Y. Wong, K. Ashton, Auto ID systems and intelligent manufacturing control, Engineering Applications of Artificial Intelligence 16 (2003) 365-376.

[8] C.Y. Wong, D. McFarlane, A. Ahmad Zaharudin, V. Agarwal, The Intelligent Product Driven Supply Chain, Cambridge Auto-ID Centre, Institute for Manufacturing, University of Cambridge (2002).

[9] M. Kärkkäinen, T. Ala-Risku, K. Främling, The product centric approach: a solution to supply network information management problems? , Computers in Industry 52 (2003) 147-159.

[10] J. Cassina, A. Cannata, M. Taisch, Development of an Extended Product Lifecycle Management through Service Oriented Architecture, Proceedings of the 1st CIRP Industrial Product-Service Systems (IPS2) conference, Cranfeild University, April 2009, pp118.

[11] W. Shen, L. M. Camarinha-Matos, R. Brennan, Special issue on multi-agent and holonic systems in manufacturing, Robotics and Computer-Integrated Manufacturing 24 (2008) 595-596

[12] S. Baïna, H. Panetto, K. Benali, A PRODUCT ORIENTED MODELLING CONCEPT Holons for systems synchronisation and interoperability, 8th International Conference on Enterprise Information Systems, ICEIS'2006.

[13] Bussmann, S., 1998, An Agent-Oriented Architecture For Holonic Manufacturing Control, First Open Workshop, Proceedings Of IMS'98 - ESPRIT Workshop In Intelligent Manufacturing Systems (Lausanne, Switzerland).

[14] A. Giret, V. Botti, Engineering Holonic Manufacturing Systems, Computers in Industry 60 (2009) 428-440

[15] A. Giret, V. Botti, Holons and agents, Journal of Intelligent Manufacturing, 15, 645-659, 2004.

[16] S. Terzi, H. Panetto, G. Morel, M. Garetti, A holonic metamodel for product traceabily in PLM, International Journal of Product Lifecycle Management 2, 3 (2007) 253-289" DOI : 10.1504/IJPLM.2007.016292.

[17] Hedden, Carole. "From Launch to Relaunch: The Secret to Product Longevity Lies in Using the Right Strategy for Each Stage of the Life Cycle." Marketing Tools. September 1997.

[18] J. Cassina, M. Tomasella, M. Marquard, A. Metin, A. Matta, M. Taisch, Development of the semantic object model for a PDKM System, International Journal of Product Development Issue (2009) 141-161.

[19] D. Kiritsis, Closed-loop PLM for intelligent products in the era of the Internet of things, Computer-Aided Design Volume 43, Issue 5, May 2011, Pages 479-501.

[20] B. Oztays, S. Baysan, F. Akpinar, Radio frequency identification (RFID) in hospitality, Technovation 29 (2009) 618-624.

[21] K. Finkenzeller, RFID Handbook: Fundamentals and Applications in Contactless Smart Cards, Radio Frequency Identification and Near-Field Communication, Third Edition. 2010 John Wiley \& Sons, Ltd.

[22] Y.F Chung, T.S Chen, M.W Lai, Efficient migration access control for mobile agents, Computer Standards \& Interfaces 31 (2009) 1061-1068.

[23] Y. Ouzrout, A. Bouras, E.H. Nfaoui, and O. El Beqqali, A Collaborative Decision-making Approach for Supply Chain Based on a Multi-agent System, Artificial Intelligence Techniques for Networked Manufacturing Enterprises Management, ISSN 1860-5168, ISBN 978-184996-118-9, Springer London Dordrecht Heidelberg New York, 2010, pp. 125-145.

[24] T.K. Shih, Mobile agent evolution computing, Information Sciences, 137 No.1, 2001, pp. 53-73.

[25] W. Shen, Q. Hao, H.J. Yoon, D.H. Norrie, Applications of agent-based systems in intelligent manufacturing: An updated review, Advanced Engineering Informatics 20 (2006) 415-431.

[26] F. Bellifemine, G. Cire , Greenwood D. Developing multi-agent systems with JADE. USA: John Wiley \& Sons, Ltd; 2006. 
[27] P. Vrba, F. Macurek, V. Marık, Using radio frequency identification in agent-based control systems for industrial applications, Engineering Applications of Artificial Intelligence 21 (2008) 331-342.

[28] IERC: IoT European Research Cluster, available on: http://www.rfid-in-action.eu/cerp-iot

[29] A. Koestler, The Ghost in the Machine, ARKANAS, 1990.

[30] P. Valckenaers, B. S. Germain, P. Verstraete, J. V. Belle, Hadeli, H. V. Brussel, Intelligent products: Agere versus Essere, Computers in Industry 60 (2009) 217-228.

[31] (DYNAMITE 2006) "Dynamic Decisions in Maintenance (DYNAMITE)" home page: http://osiris.sunderland.ac.uk/ cs0aad/DYNAMITE/Inde x.htm, accessed on 02/2012.

[32] S. Bussmann, J. Sieverding, Holonic control of an engine assembly plant-an industrial evaluation. Proc. IEEE Systems, Man, and Cybernetics Conference, Tucson, AZ, 2001.

[33] C-J Su, C-Y Wu, JADE implemented mobile multi-agent based, distributed information platform for pervasive health care monitoring, Applied Soft Computing 11 (2011) 315-325.

[34] D. Gavalas, G. E. Tsekouras, C. Anagnostopoulos, A mobile agent platform for distributed network and systems management, The Journal of Systems and Software 82 (2009) 355-371.

[35] T.C. Du, E.Y. Lib, E. Wei, Mobile agents for a brokering service in the electronic marketplace, Decision Support Systems 39 (2005) 371- 383.

[36] R. Gray, D. Kotz, S. Nog, Mobile agents for mobile computing. Technical Report PCS-TR96-285, Dartmouth
College, Department of Computer Science, Dartmouth College, 6211 Sudikoff Laboratory, Hanover, NH 03755 3510, USA, (1996).

[37] Ambient Intelligence (AmI) Technology as Enabler for Improvement, available on : http://www.ami4sme.org/

[38] ASPIRE - The EU funded project that brings RFID to SMEs, available on :http://www.fp7-aspire.eu/

[39] Building Radio Frequency IDentification for the Global Environment, available on: http://www.bridge-project.eu/

[40] 'Coordination and support action for global RFID-related activities and standardisation', available on http://cordis.europa.eu/search/index.cfm?fuseaction=new s.document\&N RCN=30283

[41] Customer in the Loop: Using Networked Devices enabled Intelligence for Proactive Customers Integration as Drivers of Integrated Enterprise available on: http://www.cuteloop.eu/

[42] Future Internet Assembly (FIA), available on: http://www.future-internet.eu/home/future-internetassembly.html

[43] IMS2020 Roadmap: Supporting Global Research for IMS Vision, available on http://www.ims.org/2011/10/ims2020-supporting-globalresearch-for-ims-vision/

[44] Promise - Product Lifecycle Management And Information Tracking Using Smart Embedded Systems, Available On: Http://Www.Promise.No/

[45] The Global RFID Interoperability Forum for Standards (GRIFS), available on: http://www.grifs-project.eu/

[46] Research Needs and Future Trends, Research in the scope of RFID and the Internet of Things, System for Mobile Maintenance Accessible in Real Time, 2008. 